\title{
Diagnostic accuracy of bedside ultrasonography in COVID-19 suspected patients admitted to the emergency department
}

\author{
Ramazan Guven ${ }^{1, \star} \oplus$, Ramazan Unal ${ }^{1}$, Burcu Genc Yavuz ${ }^{2}$, Ertugrul Ak ${ }^{1}$, \\ Gokhan Eyupoglu ${ }^{3}$, Salih Fettahoglu ${ }^{1}$, Basar Cander ${ }^{1}$
}

\author{
${ }^{1}$ Department of Emergency Medicine, \\ University of Health Sciences, Kanuni \\ Training and Research Hospital, 34303 \\ Istanbul, Turkey \\ ${ }^{2}$ Department of Emergency Medicine, \\ University of Health Sciences, \\ Haydarpasa Numune Training and \\ Research Hospital, 34668 Istanbul, \\ Turkey \\ ${ }^{3}$ Department of Emergency Medicine \\ University of Health Sciences, \\ Basaksehir Cam and Sakura City \\ Hospital, 34480 Istanbul, Turkey \\ *Correspondence \\ drramazanguven@gmail.com \\ (Ramazan Guven)
}

\begin{abstract}
Introduction: Due to the risk of cross contamination and radiation exposure of computed tomography (CT) and low sensitivity rate of X-Ray, point of care ultrasound (POCUS) lung can be used as a diagnostic tool of COVID-19 pneumonia. The current study aimed to evaluate the potential of POCUS for detection of lung pathologies caused by COVID- 19 .

Methods: This prospective observational study was conducted with 84 patients admitted to the emergency department with suspected COVID-19. CT and POCUS lung were performed for all participants. CCT was taken as the reference diagnostic method and the presence of B-lines or consolidation or pleural irregularity-thickening $(>3 \mathrm{~mm})$ in the lung in POCUS lung, were evaluated in favor of COVID-19 pneumonia.

Results: Of the 84 patients included, lesions of COVID-19 pneumonia were detected $53.5 \%$. COVID-19 pneumonia findings were shown by POCUS lung in $51.2 \%$ of participants. The left lower lobe in $48.8 \%$ and the right lower lobe in $47.6 \%$ of the patients were the most commonly affected regions. In POCUS lung, COVID-19 pneumonia lesions located in $2 \mathrm{nd}$ area for $44.0 \%$, in 7 th area for $35.7 \%$, in 8 th area for $34.5 \%$. Sensitivity of POCUS lung was found to be $88.9 \%$, specificity pointed for $92.3 \%$, positive predictive value was $93.0 \%$ and negative predictive value was $87.8 \%$.

Conclusion: POCUS lung, has a high sensitivity and specificity in the diagnosis of COVID-19 pneumonia, especially in severe lung involvement. Therefore, POCUS lung should be the method of choice as its practical use, bedside availability and avoidance of radiation exposure for COVID-19 associated lung lesions.
\end{abstract}

\section{Keywords}

COVID-19; Pneumonia; POCUS lung

\section{Introduction}

While bedside chest $\mathrm{X}$-ray provides limited diagnostic benefit in patients with pneumonia, chest computarized tomography (CCT) also has disadvantages such as high cost and the risk of transporting critically unstable patients, although the diagnostic rate is higher. Additionaly requirement for sterilization of the CCT area after each scan due to the cross infection risk of COVID-19 leads to a longer exposure time [1].

In the diagnosis of COVID-19 pneumonia, point of care ultrasound (POCUS) lung is one of the important diagnostic tools that can be used due to its cost-effectiveness, less risk of contamination, being a bedside, practical and ease of access imaging method. POCUS lung can also be used for monitoring the disease progress and evaluating the response to the treatment [2]. POCUS for the lung has been used by clinicians since the 1990s. Lichtenstein published the algorithm known as the BLUE protocol for POCUS lung in 2008
[3]. This algorithm created by using A lines from reverberation artifacts and $\mathrm{B}$ lines consisting of hyperechoic artifacts. Lichtenstein identified 2 points (upper anterior point, lower anterior point) and PLAPS (posterior lateral alveolar pleural point) point as blue points for lung pathologies. Considering data from China and especially Italy, it was seen that COVID19 pneumonia can rapidly progress to acute respiratory distress syndrome (ARDS) [4]. Soldati et al. [5] developed a method that includes 14-point lung ultrasound that provides a wider view instead of Blue-PLAPS points to diagnose and monitor COVID-19 pneumonia, which can spread to all lobes of the lung. Therefore, current study aimed to evaluate the diagnostic accuracy of POCUS Lung in COVID-19 pneumonia by using 14 points' technic, either to define which of these points were effected more by COVID-19 and its compatibility rate with CCT. 


\section{Methods}

\subsection{Study design}

This prospective convenience sample observational study was conducted to diagnose viral pneumonia with POCUS lung in patients who were classified as COVID-19 probable cases among those admitted to study center. The study center is a training and research hospital serving as one of the largest hospitals in the region during the COVID-19 pandemic. All patients included in the study, were tested for COVID-19 by real-time polymerase chain reaction (RT-PCR). The study was approved by the local ethics committee and Ministry of Health. Clinical trial registration was made with a clinicaltrials. gov ID of NCT04399681.

\subsection{Selection of participants}

Flow diagram of the study is shown in Fig. 1. Probable case definition of COVID-19 was determined in accordance with the guidelines of scientific advisory board of Turkish Ministry of Health [6]. Patients with heart failure, known chronic lung disease such as chronic obstructive pulmonary disease (COPD), chronic bronchitis, patients with lung malignancies or lung metastasis, pregnant patients and trauma patients were excluded from the study. The sample size was conducted with previous studies. We assumed that the lung ultrasound has $80 \%$ sensitivity and power of $80 \%$ with alpha $=0.05$. Thus, a minimum of 78 patients was planned to include in the study [7].

The ultrasounds performed within the scope of this study were performed by emergency physicians who had a certification of POCUS lung earned upon a bedside course of POCUS lung. The study sonographers performed POCUS lung as blinded on the patient's CCT and COVID-19 RTPCR test results. The ultrasonographic protocol consisted of scanning of the patient in the supine, right lateral decubitus, left lateral decubitis and sitting erect positions. While applying ultrasound, transducer dressed with sterile gloves covered cables, other parts of the ultrasound were sterilized with sodium hypochlorite after each use against the risk of contamination (Fig. 2). The study sonographers took personal protective measures (N95 respirator mask, surgical gown, surgical gloves, eye protection, surgical cap) while practicing.

\subsection{Interventions}

Hitachi Aloka-ProSound F37 brand ultrasonography device and 6-2 MHz Convex (UTS9123) probe and linear (UST-568) 13-5 MHz probe was used in the study. CCT was performed using Toshiba Aquilion Prime 128 slice CT scanner. Chest CT is the gold standard method used for COVID-19 pneumonia, and COVID-19 pneumonia diagnosis was made in case of typical findings (crazy-paving pattern, air bronchogram, etc.) were officially reported by radiology in favor of COVID-19.

The main hypothesis of this study is to evaluate the accuracy of lung ultrasound in diagnosing COVID-19 pneumonia. Therefore, it is more important whether the patients included in the study have a COVID-19-related pathology in their lungs rather than having positive RT-PCR tests. It was shown in previous studies that lung pathology did not develop, although RT-PCR was positive. One of the results obtained from these studies is that although the findings of COVID-19 pneumonia (ground-glass opacification) are found in CCT, the RT-PCR test can be negative $[8,9]$. The sensitivity of the RT-PCR test ranges from $50 \%-80 \%$. Therefore, it is seen that CCT is used as a reference method in COVID-19 pneumonia [10]. In accordance with the literature, the positive rate of RT-PCR among COVID-19 pneumonia patients was $66.7 \%$ in our study.

\subsection{Measurements}

CCT was performed by dividing the lung into lobes (right upper lobe, right middle lobe, right lower lobe, left upper lobe and left lower lobe) according to the official report of the radiologists. Consolidation, air bronchogram, graundglass opacities, crazy-paving pattern was sought for the interpretation of CCT in favor of COVID-19. Sonographer was asked to decide one of two conclusions of whether the patient has COVID-19 pneumonia or not. Soldati et al.'s [5] article was taken as reference and 14-point lung ultrasound, was determined (Fig. 3). Pleural movement and the presence of B lines, white lung sign, were checked for the diagnosis of pneumonia in POCUS lung. Additionally, supporting the diagnosis of pneumonia, the presence of consolidation, air bronchogram and pleural wall thickening $(>3 \mathrm{~mm})$ and its irregularity was checked (Fig. 4).

\subsection{Outcome}

The primary outcome was the sensitivity of lung ultrasound at identifying COVID-19 pneumonia against CCT as the reference standard. The secondary results of this study; to determine the presence of signs of pneumonia using 14-point lung ultrasound method.

\subsection{Statistical analysis}

All statistical analyses (sensitivity, specificity, negative predictive value, positive predictive value) were performed on MedCalc Statistical Software version v19.4.1 (MedCalc Software, Ostend, Belgium). The data of the patients are expressed as median (quartiles) for nonparametric data and percentage for categorical variables.

\section{Results}

\subsection{Characteristics of study subjects}

During the study period, 29 of 116 patients were excluded from the study since they did not meet the inclusion criteria and 3 did not complete the POCUS lung. The demographic characteristics of the participants according to the COVID-19 pneumonia status are shown in Table 1 . Of the 84 participants whose data were evaluated, $69.0 \%(\mathrm{n}=58)$ were male, the median (quartiles) age was $53.0(30.7-67.5), 31.0 \%(\mathrm{n}=26)$ was female, and the median (quartiles) age was 49.0 (31.50 63.0). When the vital signs of the patients included in the study were examined, the systolic blood pressure median (quartiles) value was $120(110-130) \mathrm{mmHg}$, the diastolic blood pressure median (quartiles) value was $70(66-80) \mathrm{mmHg}$, the median 


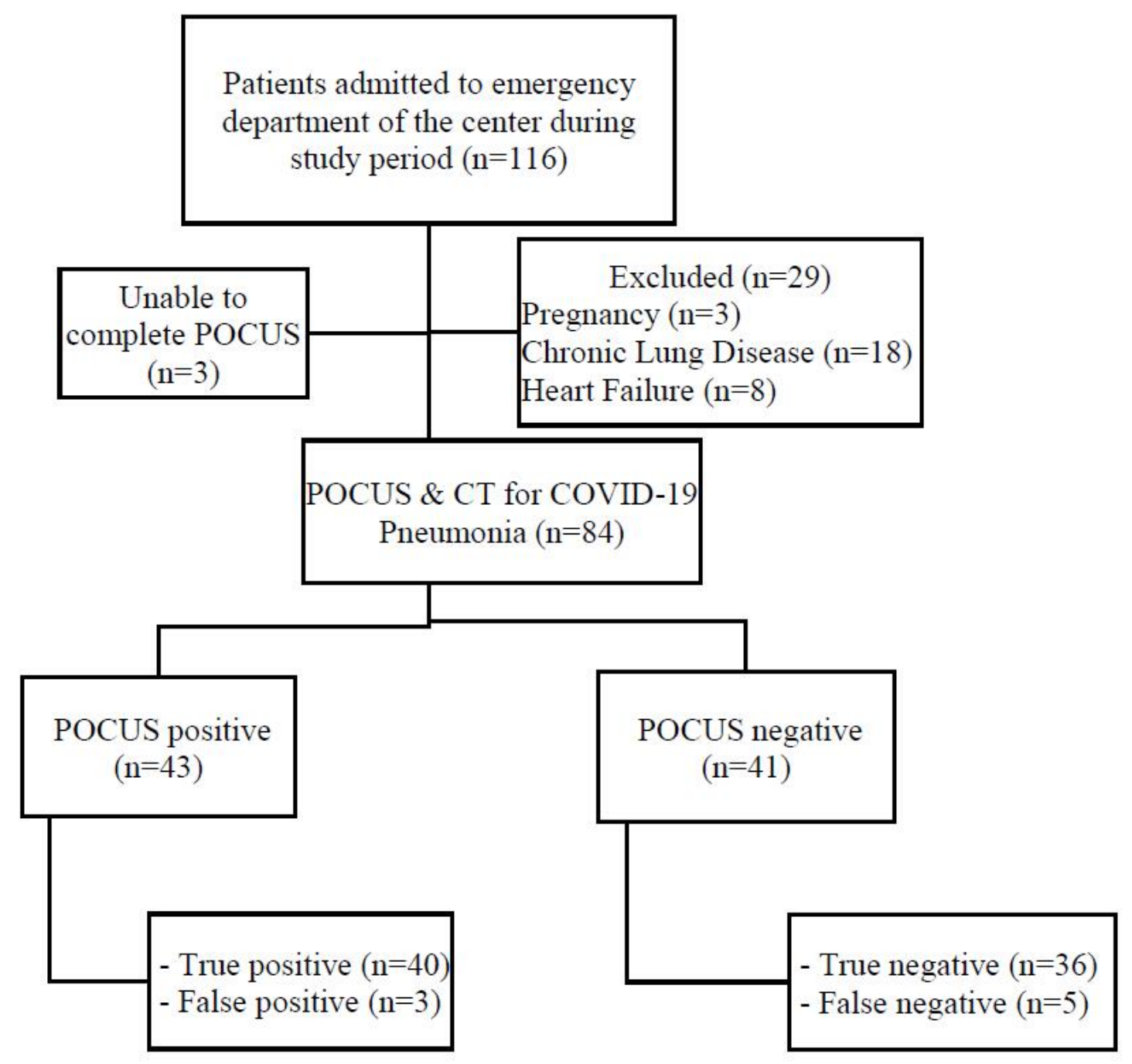

F I G U R E 1. Flowchart of the study population.

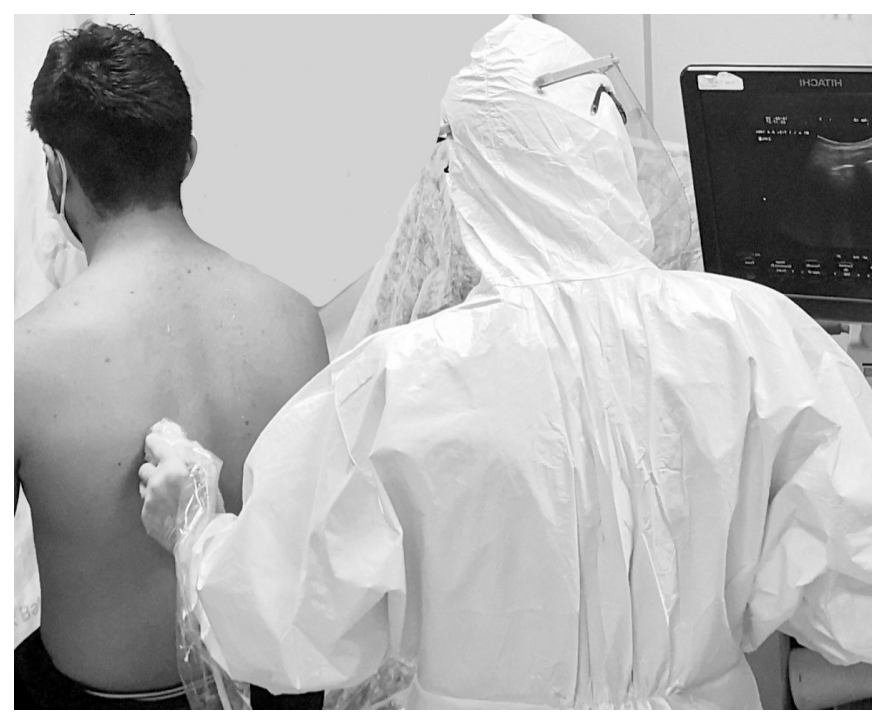

F I G URE 2. Covered cables and the probe were put in a sterile gloves, helt by sonographer.

(quartiles) value of the pulse measured per minute was 84 (7592 ), oxygen saturation median (quartiles) value was $95.5 \%$ $(92.0-97.0) .27 .4 \%(n=23)$ of the patients included in the study had hypertension, $21.4 \%(\mathrm{n}=18)$ had diabetes mellitus, and $10.7 \%(n=9)$ had coronary artery disease.

\subsection{Main results}

Lung imaging assessments are shown in Table 2. According to the CCT, $53.6 \%$ of the cases $(n=48)$ were found to have findings in favor pneumonia, and 45 of them were reported as COVID-19 pneumonia (18 mild, 14 moderate, 13 severe) and 3 patients were reported as bacterial pneumonia. Bilateral involvement was detected in $84.4 \%(n=38)$ of patients with pathological findings in favor of COVID-19 in CCT. Among imaging results reported as COVID-19 pneumonia by CCT, in order of frequency was; left lower lobe was involved in $91.1 \%$ $(\mathrm{n}=41), 88.8 \%(\mathrm{n}=40)$ had involvement in the right lower lobe, the right middle lobe was involved in $77.7 \%(\mathrm{n}=35)$, right upper lobe was involved in 51.1\% $(\mathrm{n}=23)$, left upper lobe was involved in $37.7 \%(\mathrm{n}=17)$.

According to 14-point lung ultrasound, the most common findings of COVID-19 pneumonia $(86.0 \%(n=37))$ were at the 2 nd point and then $69.7 \%(n=30)$ at the 7 th point. The most common sonographic finding of pneumonia was B-Lines with a percentage of $47.6 \%(n=40)$ (Table 2$)$.

According to POCUS lung, $51.2 \%(n=43)$ of the patients 


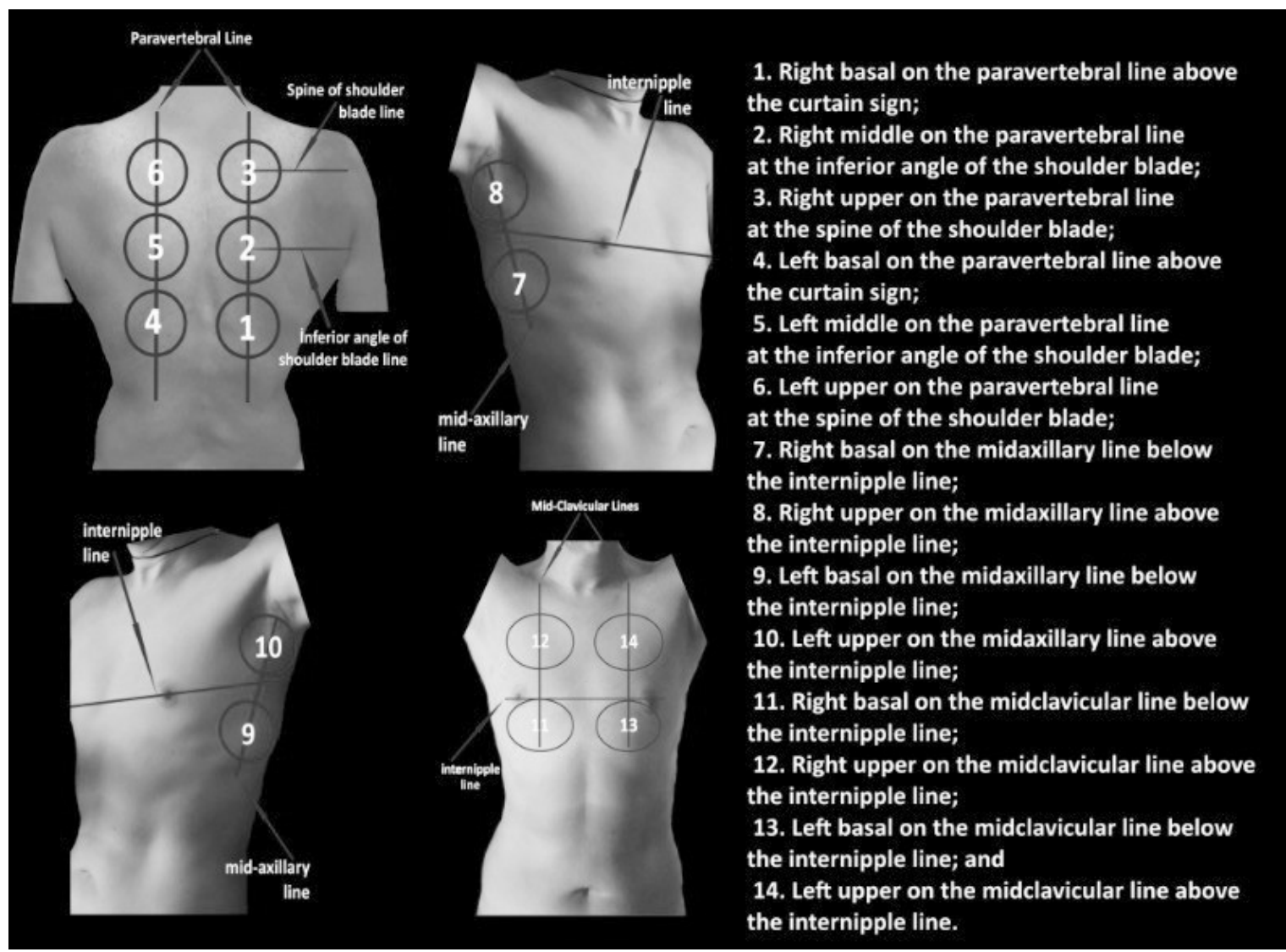

F I G U R E 3. Fourteen areas of ultrasonographic examination.

TA B L E 1. Demographic characteristics of participants.

\begin{tabular}{lcc} 
Parameter, $\mathrm{n}(\%)$ & COVID-19 pneumonia $(+)$ & COVID-19 pneumonia $(-)$ \\
\hline Sex & $(\mathrm{n}=45)$ & $(\mathrm{n}=39)$ \\
$\quad$ Female & $11(24.4)$ & $15(38.5)$ \\
$\quad$ Male & $34(75.6)$ & $24(61.5)$ \\
\hline Hypertension & $14(35.0)$ & $9(23.1)$ \\
Diabetes mellitus & $16(39.0)$ & $2(5.1)$ \\
\hline Coronary artery disease & $7(17.5)$ & $2(5.1)$ \\
Hospitalization & & $2(5.1)$ \\
$\quad$ Inpatient clinics & $20(47.6)$ & $0(0.0)$ \\
$\quad$ Intensive care unit & $14(33.3)$ & $9(23.0)$ \\
\hline Shortness of breath & $24(53.3)$ & $3(7.6)$ \\
Sore throat & $3(6.6)$ & $8(20.5)$ \\
\hline Cough & $11(24.4)$ & $19(48.7)$ \\
Fatigue & $22(48.8)$ & $7(17.9)$ \\
\hline Fever & $11(24.4)$ & \\
\hline
\end{tabular}

were evaluated as COVID-19 pneumonia. However, POCUS lung was found as false positive in 3 patients and false negative in 5 patients. Accordingly, the sensitivity of POCUS lung was calculated as $88.9 \%$, specificity $92.3 \%$, positive predictive value $93.0 \%$, negative predictive value $87.8 \%$ (Table 3 ).

\section{Discussion}

CXR and CCT are used to detect the presence of lung pathology due to COVID-19 and to see its severity. However, due to the known disadvantages of CXR (low sensitivity rate) and $\mathrm{CT}$ (radiation risk, possibility of cross contamination), POCUS lung, which is an alternative diagnosis method, started to be used especially in the later stages of the pandemic $[1,2,11]$. 


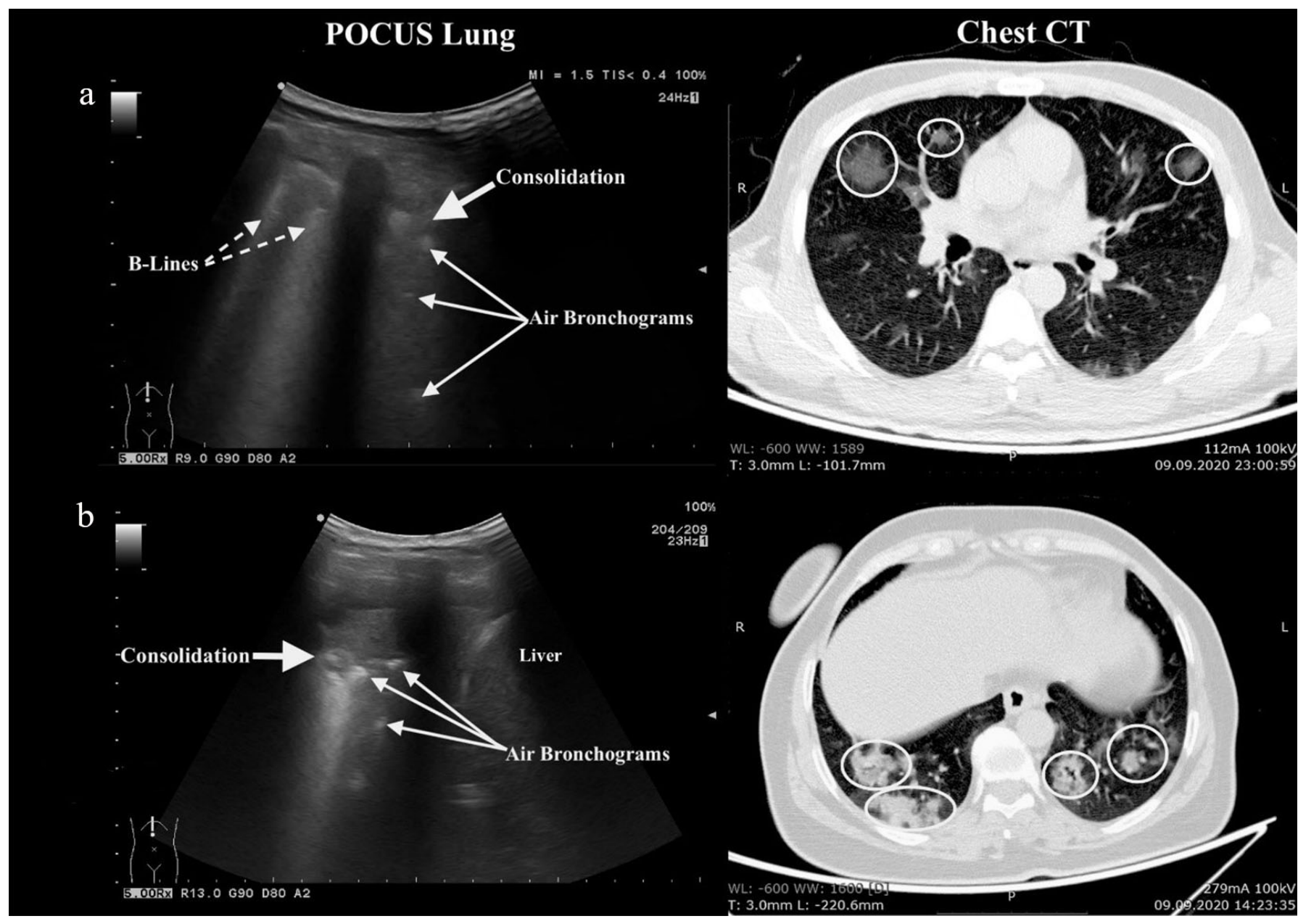

F I G U R E 4. Imaging features of COVID-19 patients. (a) Solid yellow arrow-signed consolidation and dashed yellow arrows showed nonconfluent B-lines in 2nd area of POCUS lung. Areas surrounded by yellow circles demonstrated ground glass opacity with consolidation in upper right lobe on CCT. (b) Solid yellow arrow showed consolidation with air bronchograms pointed by multiple yellow arrows in 1st area of POCUS lung. Yellow circles surrounded multiple ground glass opacities with consolidations in both lungs on CCT.

This study revealed that POCUS lung had $88.9 \%$ sensitivity and $92.3 \%$ specificity when CCT was taken as reference in detecting lung pathology due to COVID-19 infection. Throughout the study, POCUS lung was more accessible and allowed bedside application compared with CCT. BLUE and PLAPS points are used for sonography to detect lung pathologies. However, COVID-19 pneumonia with its widespread lung involvement, would be better to be evaluated by covering more areas. Therefore, the POCUS lung protocol in 14-point recommended by Soldati et al. [5] for COVID-19 pneumonia, which provides a more detailed view to us, constituted the field of view of our study. COVID-19 pneumonia affects the middle and lower lobes in the right lung and the lower lobes in the left lung more frequently [12]. Similarly, CCT results in the current study, demonstrated that $41.7 \%$ of the patients had involvement in the right middle lobe, $47.6 \%$ in the right lower lobe, and $48.8 \%$ in the left lower lobe. Looking at the POCUS lung examination points, $44.0 \%$ of the patients had pathology in the POCUS 2nd point and $34.5 \%$ in the POCUS 5th point. The reason for detecting less pathology in POCUS 3th point and POCUS 6th point in the POCUS lung is due to the upper lobe involvement of COVID-19 infection, similar to the results of CCT. Detection of less pathology in POCUS 11 th point, POCUS 12th point, POCUS 13th point and POCUS 14th point can be explained with involving the anterior region of the lung is less in COVID-19 pneumonia. Additionally, due to the presence of the heart image in the POCUS 13th point and POCUS 14th point, pathology becomes less detectable in these areas. The POCUS Lung and CCT results of present study are consistent with the literature and it is seen that the posterior regions of the lower lobes of the lung are mostly affected [13]. A limited number of articles exist on POCUS lung showing COVID-19 pathologies, most of them are technical reports and narrative reviews. Of these, Moore S et al. [14] suggested that since POCUS lung had high sensitivity and specificity, and that the use of systemic ultrasound will be beneficial not only in emergency services but also in intensive care units (ICU), in the follow-up of critical COVID-19 patients, thus reducing the reuse of radiographic imaging methods. A study, in which POCUS lung used in demonstrating and monitoring lung pathologies in COVID-19 ICU patients, $100 \%$ of the patients had B-lines (confluent or separate) and $61.7 \%$ of them had consolidation at their first sonographic examinations on admittance, and these findings were reported to be significantly 
TA B L E 2. Findings on POCUS lung for COVID-19 pneumonia, frequency rate of location of COVID-19 pneumonia-positive areas of fourteen lung points.

\begin{tabular}{|c|c|c|}
\hline POCUS, n (\%) & COVID-19 pneumonia $(+)$ & COVID-19 pneumonia (-) \\
\hline & $(n=45)$ & $(n=39)$ \\
\hline \multicolumn{3}{|l|}{ POCUS lung } \\
\hline Pneumonia & 43 ( 5 false positive) & 41 (3 false negative) \\
\hline B-Lines & 40 & 3 \\
\hline Consolidation & 22 & 3 \\
\hline Pleural irregularity-thickening & 29 & 2 \\
\hline \multicolumn{3}{|l|}{ 14-Point lung ultrasound } \\
\hline 1. Point (Right basal on the paravertebral line) & 17 & 0 \\
\hline 2. Point (Right middle on the paravertebral line) & 37 & 0 \\
\hline 3. Point (Right upper on the paravertebral line) & 16 & 0 \\
\hline 4. Point (Left basal on the paravertebral line) & 21 & 0 \\
\hline 5. Point (Left middle on the paravertebral line) & 29 & 0 \\
\hline 6. Point (Left upper on the paravertebral line) & 14 & 0 \\
\hline 7. Point (Right basal on the midaxillary line) & 30 & 2 \\
\hline 8. Point (Right upper on the midaxillary line) & 29 & 0 \\
\hline 9. Point (Left basal on the midaxillary line) & 19 & 1 \\
\hline 10. Point (Left upper on the midaxillary line) & 18 & 0 \\
\hline 11. Point (Right basal on the midclavicular line) & 16 & 0 \\
\hline 12. Point (Right upper on the midclavicular line) & 19 & 0 \\
\hline 13. Point (Left basal on the midclavicular line) & 12 & 0 \\
\hline 14. Point (Left upper on the midclavicular line) & 14 & 0 \\
\hline
\end{tabular}

TA B L E 3. Test characteristics of POCUS lung in diagnosing COVID-19 pneumonia.

\begin{tabular}{|lcc|}
\hline & Value & $95 \%$ CI \\
\hline Sensitivity & $88.9 \%$ & $75.95 \%-96.29 \%$ \\
\hline Specificity & $92.3 \%$ & $79.13 \%-98.38 \%$ \\
\hline Positive likelihood ratio & 11.5 & $3.88-34.44$ \\
\hline Negative likelihood ratio & 0.12 & $0.05-0.28$ \\
\hline Positive predictive value & $93.0 \%$ & $81.73 \%-97.55 \%$ \\
\hline Negative predictive value & $87.8 \%$ & $75.8 \%-94.3 \%$ \\
\hline
\end{tabular}

regressed in the week of discharge [15]. In our study, there was B-lines and consolidation, either, in $100 \%$ of 14 patients hospitalized in ICU while B-lines were present in $86.4 \%$ of patients hospitalized in pandemic ward $(\mathrm{n}=22)$, and consolidation in $70 \%$ of them. In this study, the scoring system or any classification was not used because of the CCT reports were reported as "in favor of COVID-19" or "COVID-19 pneumonia was not considered". In a study on asymptomatic COVID-19 patients, POCUS lung detected the pneumonia findings of US with $66.7 \%$ sensitivity, $100 \%$ specificity, $100 \%$ positive predictive value, $85.71 \%$ negative predictive value [16]. Among all patients in the current study, the sensitivity of POCUS lung was found to be $88.9 \%$, specificity $92.3 \%$, positive predictive value was $93.0 \%$, and negative predictive value was $87.8 \%$. All of the false positive cases were reported as bacterial pneumonia in CCT. All of those, who were evaluated as false negative, were patients with mild pneumonia (unifocal opacity). Lu et al. [17] reported that the sensitivity of lung US for COVID-19 was $68.8 \%, 77.8 \%, 100 \%$, and the specificity was $85.7 \%, 76.2 \%$, $92.9 \%$, respectively. Similarly, the present study revealed that as the severity of the disease increases, the diagnostic power of POCUS lung increases, so that all intensive care patients (n $=14$ ) were correctly diagnosed with POCUS lung.

\section{Limitations}

This study has several limitations. Although it is the largest pandemic hospital in the region, the first limitation of this study is that it cannot be generalized with other hospitals because it is a single center. Despite ultrasound (USG) training is included in the curriculum of the emergency medicine clinic in our country, currently, the USG training of all emergency physicians, especially the POCUS lung training, is not at the desired level. The fact that USG is a subjective tool, also directly affects the sensitivity and specificity of POCUS lung. Another limitation of the study was the fact that the study directors were working in 24-hour shifts, which led to the exclusion of patients outside of their switch.

There were also some limitations due to the current disease state. First, since this study was conducted on COVID-19, which has a high risk of contagion, sonographers had difficulty to have the appropriate positions. Second, the use of only one 
USG device for the study and the time required to sterilize this USG device after each examination caused some patients who came in the mean time to be excluded from the study. Third, in case of lung pathology in patients with suspected COVID-19 infection, they clinically give sonographers some clues (e.g., tachypnea, tachycardia, high fever). This seems to cause an increase in accuracy in favor of USG.

\section{Conclusions}

POCUS lung, with fourteen visual fields, provides sufficient diagnostic opportunity. Although POCUS lung is not at the desired level in distinguishing false-negativity risk and bacterial pneumonia from COVID-19 pneumonia in patients with mild pneumonia, it has a high level of sensitivity and specificity in severe COVID-19 pneumonia. Therefore, POCUS lung can be used as the method of choice due to its practical use, bedside availability and avoidance of radiation exposure for COVID19 associated lung lesions.

\section{AUTHOR CONTRIBUTIONS}

RG, GE, RU and BC designed this study. RG, SF, BGY, EA, RU and GE, supervised the overall data collection process, had full access to all the data in the study, and takes responsibility for the integrity of the data. $\mathrm{RG}$ and GE conducted the data analysis. RG, EA, SF, BGY and BC wrote the initial draft of the article. All authors provided substantial review and feedback on the final version of the article. RG takes responsibility for the paper as a whole.

\section{ETHICS APPROVAL AND CONSENT TO PARTICIPATE}

All participants were included in the study following their approval of written informed consent. The study was approved by the local ethics committee (Ethics Committee approval number: KAEK/2020.03.50) and Ministry of Health. Clinical trial registration was made with a clinicaltrials.gov ID of NCT04399681.

\section{ACKNOWLEDGMENT}

The authors thanks to all emergency department staff for their contribution during data collection.

\section{FUNDING}

This study received no external funding.

\section{CONFLICT OF INTEREST}

The authors declare no conflict of interest.

\section{AVAILABILITY OF DATA AND MATERIAL}

The datasets generated during and/or analysed during the current study are available from the corresponding author on reasonable request.

\section{REFERENCES}

[1] Li M. Chest CT features and their role in COVID-19. Radiology of Infectious Diseases. 2020; 7: 51-54.

[2] Fiala MJ. A Brief Review of Lung Ultrasonography in COVID-19: is it Useful? Annals of Emergency Medicine. 2020; 75: 784-785.

[3] Lichtenstein DA, Mezière GA. Relevance of lung ultrasound in the diagnosis of acute respiratory failure: the BLUE protocol. Chest. 2008; 134: 117-125.

[4] Remuzzi A, Remuzzi G. COVID-19 and Italy: what next? Lancet. 2020; 395: $1225-1228$.

[5] Soldati G, Smargiassi A, Inchingolo R, Buonsenso D, Perrone T, Briganti $\mathrm{DF}$, et al. Proposal for International Standardization of the Use of Lung Ultrasound for Patients With COVID-19: A Simple, Quantitative, Reproducible Method. Journal of Ultrasound in Medicine. 2020; 39: 1413-1419.

[6] Scientific Advisory Board Republic of Turkey Ministry of Health. SARS-CoV-2 Infection: general information, epidemiology and diagnosis April 21, 2020. 2020. Available at: https: //covid19bilgi.saglik.gov.tr/depo/Sunumlar/COVID-19Epidemiyoloji-Tani-Tedavi.pdf (Accessed: 25 May 2020).

[7] Haak SL, Renken IJE, Jager LC, Lameijer H and Kolk BYM. Diagnostic accuracy of point-of-care lung ultrasound in COVID-19. Emergency Medicine Journal. 2021; 38: 94-99.

[8] Fang Y, Zhang H, Xie J, Lin M, Pang P, Ji W. Sensitivity of Chest for COVID-19: Comparison to RT-PCR. Radiology. 2020; 296: E117.

[9] Korkmaz I, Dikmen N, Keles FÖ, Bal T. Chest CT in COVID-19 pnumonia: correlations of imaging findings in clinically suspected but repeatedly RT-PCR test-negative patients. Egyptian Journal of Radiology and Nuclear Medicine. 2021; 52: 96.

[10] Ai T, Yang Z, Hou H, Chenao Z, Chen C, Lv W, et al. Correlation of Chest CT and RT-PCR Testing in Coronavirus Disease 2019 (COVID19) in China: A Report of 1014 Cases. Radiology. 2020; 296: E32-E40.

[11] Cleverley J, Piper J, Jones MM. The role of chest radiography in confirming covid-19 pneumonia. British Medical Journal. 2020; 370: $\mathrm{m} 2426$.

[12] Li Y, Xia L. Coronavirus Disease 2019 (COVID-19): Role of Chest CT in Diagnosis and Management. American Journal of Roentgenology. 2020; 214: $1280-1286$.

[13] Zhao W, Zhong Z, Xie X, Yu Q, Liu J. Relation between Chest CT Findings and Clinical Conditions of Coronavirus Disease (COVID-19) Pneumonia: a Multicenter Study. American Journal of Roentgenology. 2020; 214: 1072-1077.

[14] Moore S, Gardiner E. Point of care and intensive care lung ultrasound: a reference guide for practitioners during COVID-19. Radiography. 2020; 26: e297-e302.

[15] Alharthy A, Faqihi F, Abuhamdah M, Noor A, Naseem N, Balhamar A, et al. Prospective Longitudinal Evaluation of Point-of-Care Lung Ultrasound in Critically Ill Patients With Severe COVID-19 Pneumonia. Journal of Ultrasound in Medicine. 2020; 40: 443-456.

[16] Haidan LM, Bingqi ZM, Haiyan KM, Yuanyuan ZM, Keyan LM, Dudu WM, et al. Application Value of Lung Ultrasound in Asymptomatic Patients with Confirmed COVID-19. Advanced Ultrasound in Diagnosis and Therapy. 2020; 4: 67.

[17] Lu W, Zhang S, Chen B, Chen J, Xian J, Lin Y, et al. A Clinical Study of Noninvasive Assessment of Lung Lesions in Patients with Coronavirus Disease-19 (COVID-19) by Bedside Ultrasound. Ultraschall in Der Medizin—European Journal of Ultrasound. 2020; 41: 300-307.

How to cite this article: Ramazan Guven, Ramazan Unal, Burcu Genc Yavuz, Ertugrul Ak, Gokhan Eyupoglu, Salih Fettahoglu, et al. Diagnostic accuracy of bedside ultrasonography in COVID-19 suspected patients admitted to the emergency department. Signa Vitae. 2022;18(1):55-61. doi:10.22514/sv.2021.116. 